\title{
Der Mesmerismus im deutschen Geistesleben
}

\author{
Von Walter Artelt, Frankfurt am Main
}

Unser Wissen um die Natur ist bis in das 19. Jahrhundert hinein von den Angehörigen aller nur möglichen Stände und Berufe zusammengetragen worden. Demgegenüber war die Medizin von jeher eine esoterische Wissenschaft. Der Schwerpunkt ihrer Literatur lag zu allen Zeiten bei denen, deren Beruf das Heilen war. Nur einmal in den letzten Jahrhunderten schien es so, als ob die ständisch-fachliche Umzäunung auf weite Strecken hin gefallen und jedermann, der sich aufgerufen fühlte, auch berufen sei mitzuwirken: in den ersten Jahrzehnten des Mesmerismus. Der Mesmerismus war weder in der Idee noch in der Praxis etwas grundsätzlich Neues. Vieles war längst vor MESMER ausgesprochen worden, und auch die «magnetischen» Streichungen der Mesmeristen haben eine lange und interessante Vorgeschichte. Mesmer entwickelte diese Ansätze zu einer in sich geschlossenen Lehre von bestechender Folgerichtigkeit, die alle früheren Erfahrungen auf einfachste Weise zu erklären schien. So kam es unter dem Einfluß seiner Persönlichkeit und seiner Erfolge zu jener Bewegung, die als «Mesmerismus» immer weitere Kreise in ihren Bann zog. Die Zeitumstände taten das ihre. Schon seit Jahrzehnten waren viele Kräfte am Werk, die zweite geheimnisvolle Naturkraft, die Elektrizität, die unsichtbar war und sichtbar wirkte wie der Magnetismus, zu erklären, zu beherrschen und - auch für die Medizin - zu nutzen. Aber die therapeutischen Möglichkeiten der Elektrizität schienen eng beschränkt: man suchte Lähmungen und Rheumatismus zu heilen, machte elektrische Wiederbelebungsversuche und erwog die Anwendung elektrischer Schläge anstelle von Aderlässen. Nicht viel weiter war der Indikationsbereich des Eisenmagneten, dem man Heilwirkungen bei Neuralgien, Rheumatismus, Augenleiden, Krämpfen und Koliken zuschrieb. Um so großartiger waren die Perspektiven, die sich eröffneten, wenn man jetzt mit Mesmer den Magnetismus des Magneteisens nur als eine besondere Erscheinungsform eines magnetischen Fluidums betrachtete, das das Weltall erfüllt, auch im Menschen wirksam ist und von Mensch zu Mensch übertragen werden kann.

Das Gros der Ärzte blieb freilich zunächst ablehnend. Es witterte Scharlatanerie und Betrug, auch da, wo ehrliches Bemühen unverkennbar ist. Aber der Mesmerismus gewann schnell viele überzeugte Anhänger in Laienkreisen. So stand auch bei der Einführung des Mesmerismus in Deutschland 
nicht ein Arzt Pate, sondern ein Prediger in Zürich: Johann Caspar Lavater. Lavater geriet 1785 in den Bann des Mesmerismus und behandelte nach den Anweisungen des Genfer Arztes Butisi seine kranke Frau mit magnetischen Streichungen. Er wurde nun zum begeisterten Künder des Mesmerismus, schrieb enthusiastische Briefe auch an seine Freunde in Deutschland und beschwor sie, sich mit dem tierischen Magnetismus zu befassen ${ }^{1}$. Der Markgraf Karl Friedrich in Karlsruhe, den Lavater für den Mesmerismus interessierte, entsandte daraufhin einige Männer seiner Umgebung zum Studium des tierischen Magnetismus in das nächste Zentrum der Mesmeristen, nach Straßburg, unter ihnen den Mathematiker und Physiker BöckmanN ${ }^{2}$.

In Straßburg stand - neben den Ärzten OSTERTAG und WüRTZ - wieder ein Laie im Mittelpunkt des Mesmeristenkreises, der Artilleriekapitän Marquis de Puységur. Böckmann wurde ein eifriger Anhänger der Lehre MESMers und gab 1787 ein «Archiv für Magnetismus und Somnambulismus» heraus. 1788 besuchte Mesmer selber Karlsruhe. Von Böckmann und Puységur ließ sich wiederum Eberhard Gmelin, Amtsarzt in Heilbronn, in die Geheimnisse des Mesmerismus einführen. Auch in der Residenzstadt Mannheim, die durch die kurpfälzische Akademie zu einem geistigen Zentrum geworden war, entwickelte sich um diese Zeit eine «Magnetisir-Loge», in der der Intendant des Mannheimer Theaters, W.H.von Dalberg, lebhaft mitwirkte ${ }^{3}$.

Eine weitere Keimzelle des Mesmerismus entstand - wieder durch LAVATER ins Leben gerufen - in Bremen. LAVATER war im Sommer 1786 eine Woche in der Stadt Bremen, die sich vergeblich bemüht hatte, ihn als Prediger zu gewinnen, und heilte dort die achtzehnjährige Tochter eines Kaufmannes, die an «Krämpfen und Konvulsionen» litt, durch magnetische Behandlung. So wurden der behandelnde Arzt des Mädchens, Wienнolt, und sein Kollege OLBers, der zu den bedeutendsten Astronomen seiner Zeit gehört, zu Anhängern Mesmers. Heineken und Treviranus schlossen

${ }^{1}$ Vgl. J. C.LAVATERS bisher ungedruckte Briefe und Aufsätze über den thierischen Magnetismus, mitgetheilt von D.G. Kieser, in Arch. thier. Magn., hg. von EschenMaYer, Kreser und NeES von EsenBecK, Bd. 8 (1821), III, S. 1-59, und Bd. 9 (1821), I, S. 1-60.

${ }^{2}$ Vgl. dazu, wie auch zum folgenden, Rudolf Tischner und KarL Bittel, Mesmer und sein Problem, Stuttgart 1941.

${ }^{3}$ Vgl. Adolf Kistner, Die Pflege der Naturwissenschaften in Mannheim zur Zeit Karl Theodors (= Geschichte der Kurpfälzischen Akademie der Wissenschaften in Mannheim, hrsg. vom Mannheimer Altertumsverein, Bd. 1), Mannheim 1930, S. 76. 
sich ihnen an ${ }^{4} .1787$ begann ein «Magnetisches Magazin für Niederdeutschland» in Bremen zu erscheinen.

Noch mancher tummelte sich um diese Zeit auch in Deutschland auf dem Felde des tierischen Magnetismus, wie der Straßburger Dufour, der mit seinen Vorlesungen in Breslau einen Arzt, einen Leutnant, einen Kaufmann und einen Propst für den Mesmerismus gewann ${ }^{4 a}$, und der Graf von Thun, der in den neunziger Jahren in Leipzig als Magnetiseur von sich reden machte ${ }^{5}$.

Über Mesmer und die kleine Schar seiner Anhänger ergoß sich eine ganze Flut von Hohn und Spott in der deutschen medizinischen und nichtmedizinischen Literatur. Darunter war der Erstlingsaufsatz eines jungen Arztes, der bald zum engeren Kreis um GoEthe in Weimar gehören sollte, HufeLANDS Artikel «Mesmer und sein Magnetismus» in WiELANDS «Teutschem Merkur» vom Jahre 1784. Mesmer sei, so heißt es da, «höchst wahrscheinlich ein sich selbst betrügender Schwärmer, sein Magnetismus ein Hirngespinst und seine Wunder Wirkungen kranker Einbildungskraft». Der junge Philologe Friedrich August Wolf benutzte 1787 einen Vortrag über die Geschichte des «magnetischen Somnambulismus im Altertum» dazu, zugleich mit dem Heilbetrieb der antiken Asklepieien Lavater und den Mesmerismus zu treffen ${ }^{6}$. In der schönen Literatur verspottete Moritz August von Thümmel in seiner Reise in die mittäglichen Provinzen von Frankreich im Jahre 1785-86 (1791 ff.) den Mesmeristenzirkel in Straßburg; «die Leichtgläubigkeit eines Kranken ist der verzeihlichste Glaube»!?

Nur der Dichter des Don Carlos, der 1780 sein Medizinstudium mit einer Probeschrift Über den Zusammenhang der tierischen Natur des Menschen mit seiner geistigen abgeschlossen hatte, lauschte schon damals nachdenklich und nicht ohne Sympathie auf die Stimme der Mesmeristen; Caroline von Wolzogen berichtet vom Jahr 1793 über Schiller: «Im September besuchte ich Schillers in Heilbronn ... Ich erinnere mich sehr merkwürdiger Gespräche, die Schiller in Heilbronn mit dem berühmten Arzte

${ }^{4}$ Vgl. H.TJAden, Bremen und die bremische Ärzteschaft seit dem Beginne des 19. Jahrhunderts, Bremen 1932, S. 72-4.

4a Vgl. Friedrich v. Oppeln-Bronikowski, David Ferdinand Koreff, Serapionsbruder, Magnetiseur, Geheimrat und Dichter, Berlin-Leipzig (1928), Einleitung S. 15 f., Anm. 2.

5 Vgl. Kurt Sprenger, Kritische Übersicht des Zustandes der Arzneykunde in dem letzten Jahrzehend, Halle 1801, S. 217.

6 Vgl. Wilhelm Erman, Der tierische Magnetismus in Preußen vor und nach den Freiheitskriegen (= Beiheft 4 der Hist. Z.), München und Berlin 1925, S. 14f.

7 Sämmtliche Werke, Bd. 1, Leipzig 1853, S. 72. 
GMELIN über tierischen Magnetismus führte. Diese wichtige Entdeckung unserer Zeit zog ihn sehr an; doch fand er seinen eigenen Krankheitszustand für Versuche mit dieser Heilmethode nicht geeignet.» ${ }^{8}$

Die Stimmen der Nachdenklichen aber übertönte der Chor der lauten Spötter, die da glaubten, die Phänomene des Mesmerismus leichthin als Hysterie, Betrug oder Selbstbetrug abtun zu können. So sah ihn, an der Jahrhundertwende zurückschauend, auch der Medizinhistoriker Sprengel, der 1801 in seiner Kritischen Übersicht des Zustandes der Arzneykunde in dem letzten Jahrzehend schrieb: «In der Tat schien der tierische Magnetismus, nach den Aussagen einiger Freunde desselben, den Weg in das wahre Eldorado der überirdischen Wissenschaften zu eröffnen, wohin ein jeder, der nur Glauben genug habe, durch einige angenehme Manipulationen an Brust, Herzgrube und Lenden, ohne alle weiteren Vorkenntnisse, versetzt werden könne. $)^{9}$ SpREngel tat also selbèr gerade das, weswegen er einige Dutzend Seiten später seinerseits Schelling so bitter tadelte: «Ein billiger Kenner seiner Wissenschaft läßt einem jeden gern sein Spielwerk; aber Unduldsamkeit und Hohn, mit Unkunde der empirischen Dinge, das erträgt sich nicht leicht.» ${ }^{10}$ SPRENGEL verkannte die geistige Situation der Zeit um die Jahrhundertwende. Ein tiefgreifender Wandel hatte sich angebahnt. Seine in der gleichen Kritischen Übersicht ausgesprochene Ansicht, daß Schelling eine Zeitlang der Herold der Naturphilosophie gewesen sei und daß die spekulative Physiologie keine lange Dauer verspreche ${ }^{11}$, erwies sich als falsch. Die romantische Naturphilosophie beherrschte in Deutschland immerhin für die Dauer einer Generation das Feld. Und mit der Romantik änderte sich das geistige Klima für den Mesmerismus in Deutschland von Grund auf.

Ahnung und innere Schau waren die Triebkräfte des romantischen Denkens; ihr Ziel, die Seele «als das Unendliche des Leibes» (Steffens) zu fassen. «Nach innen geht der geheimnisvolle Weg», so sprach Novalis aus, was sie alle bewegte, und «Wo ist die Seele meiner Seele?» fragte Friedrich Schlegel. So beschäftigten der magnetische Rapport zwischen zwei Menschen, der magnetische Schlaf, Somnambulismus und Hellsehen die Romantiker immer von neuem. Gerade im Unbewußten suchte man

\footnotetext{
${ }^{8}$ Caroline von Wolzogen, geb. von Lengefeld, Schillers Leben, 2. Auflage, Stuttgart 1845 , S. 238.

9 Sprengel, a.a. O., S. 54.

10 Ebenda, S. 326.

11 Ebenda, S. 326 und 483.
} 
den Schlüssel zur Erkenntnis des Seelenlebens, zum Urgrund des Lebens, zum Absoluten, das die Seele im somnambulen Zustande schaut (Novalis).

Damit hörte der Mesmerismus vollends auf, eine nur medizinische Lehre zu sein, ja die Medizin selber schien sich als selbständige Wissenschaft aufzulösen. «Der Name Medizin», so sprach der Arzt K. E. Schelling, der Bruder des Philosophen, es 1807 aus, «begreift so vieles in sich, daß es schwer ist, von ihr in abstracto zu reden, außerdem läuft ihr höherer oder niedriger Stand so sehr al pari mit dem der Philosophie, Physiologie u.s.f., daß von ihr als einer selbständigen Wissenschaft eigentlich die Rede gar nicht sein kann».12 Ebenso einmütig, wie Medizin, Geisteswissenschaft und schöne Literatur zuvor die Lehre Mesmers bekämpft hatten, wirken nun Ärzte, Philosophen und Dichter nebeneinander und miteinander für den Mesmerismus. Die Philosophen untersuchen die medizinischen Möglichkeiten des tierischen Magnetismus, Ärzte philosophieren über die Zustände des Unbewußtseins. Es ist kein Zufall, daß Jung-Stilling, der 1808 seine auf den Mesmerismus begründete Theorie der Geisterkunde veröffentlichte, längst aufgehört hat, Arzt zu sein, daß Gotthilf Heinrigh Schubert, der um die gleiche Zeit seine Vorlesungen über die Ansichten von der Nachtseite der Naturwissenschaften in Dresden hielt und 1808 publizierte, 1809 in den Schuldienst hinüberwechselte.

Es sind entscheidende Jahre für den Mesmerismus. Schuberts Vorlesungen wirkten tief und nachhaltig auf das geistige Leben in Deutschland ein. Hier lernte HeInRIGH von KLEIST jene Krankengeschichte kennen, in der Eberhard Gmelin die Geschichte einer magnetischen Schlafwandlerin, der Lisette Kornmacher aus Heilbronn, erzählte. 191 magnetische Sitzungen verzeichnet GMELINs Bericht, eine jener schier endlosen, umständlichweitschweifigen Krankengeschichten, die so kennzeichnend für die Literatur des Mesmerismus sind. Unter dem unmittelbaren Eindruck dieser Krankengeschichte schrieb KLEIST sein Käthchen von Heilbronn, das mit dem Grafen vom Strahl auf eine «magnetische» Weise verbunden ist. Auch E.T.A.Hoffmann las Schuberts Ansichten und gab 1813, wieder in Dresden, den Schubertschen Ideen in seinem «Goldenen Topf» dichterischen Ausdruck. Im gleichen Jahr war auch seine Novelle Der Magnetiseur entstanden.

Der Einfluß des Mesmerismus auf die romantische Dichtung ist jetzt auf Schritt und Tritt spürbar. ARNim schildert in seiner Gräfin Dolores (1810)

${ }^{12}$ K. E.ScheLLING, Ideen und Erfahrungen über den thierischen Magnetismus, in: Jahrbücher der Medicin als Wissenschaft, hg. von A.F.MARcus und F.W.Schelling, Bd.2, Tübingen 1807, S. 27. 
die Figur eines Magnetiseurs: «Der Markese hatte sich alle Geheimnisse der Rosenkreuzer angeeignet, um sie, vermischt mit dem Mesmerschen Magnetismus, als eine furchtbare Geisterhand in das Innerste der Gemüter auszustrecken.» Durch «metallne Scheiben» wird die tote Biondette in Brentanos Romanze vom Rosenkranz wiedererweckt. Nur in den Novellen TiEcks erscheint der Mesmerismus als eine Sünde wider die Natur oder gar als lächerlicher Unfug ${ }^{13}$.

Auch Goethes Wahlverwandtschaften, die 1809 erschienen, gehen an dem Phänomen des Mesmerismus nicht vorüber. Aber GoEthE war kein Romantiker, er distanzierte sich behutsam: Ottilie ist als ein für okkulte Kräfte besonders empfängliches Wesen geschildert, das über einem unterirdischen Kohlenlager von Kopfschmerzen befallen wird. Der Begleiter des Lords nimmt diese Beobachtung zum Anlaß, Experimente mit dem siderischen Pendel an Ottilie zu machen. Goethe stützte sich bei dieser Schilderung auf die Versuche, die Schelding, BaAder und Ritter 1806 in München mit dem italienischen «Erz- und Wasserfühler» CAмpetri durchgeführt hatten. Schellings Bericht darüber in der «Jenaischen Allgemeinen Literaturzeitung» von 1807 hatte ausdrücklich auf die Zusammenhänge des «Erz- und Wasserfühlens» mit dem Mesmerismus hingewiesen; er glaube, so heißt es da, daß «dieses ganze Phänomen sich auflösen werde in jene tief verkannte, aber bald nicht länger zu verkennende Erscheinung, die seit einigen Jahrzehnten unter dem Namen des tierischen Magnetismus so verschiedene Schicksale gehabt hat». So läßt auch Goethe den Begleiter des Lords, als Ottiliens Kopfschmerzen sich bei den Pendelversuchen wieder einstellen, versichern, «daß er sie von diesem Übel völlig heilen wolle, wenn sie sich seiner Kurart anvertraue. Man war einen Augenblick ungewiß; Charlotte aber, die geschwind begriff, wovon die Rede sei, lehnte den wohlgemeinten Antrag ab, weil sie nicht gemeint war, in ihrer Umgebung etwas zuzulassen, wovor sie immerfort eine starke Apprehension gefühlt hatte.» Auch Goethes Leser verstanden, auf welche besondere Kurart hier solcherart angespielt wurde, den Mesmerismus. Charlottens Reaktion aber gab GoEthes eigene Gefühle wieder. «Nie werde ich vergessen», so hat uns $\mathrm{Hu}$ FELAND später rückschauend berichtet, «was mir einst GöTHE bei einer Unterredung über diesen Gegenstand sagte: ,Ich habe mich nie mit dem Magnetismus befassen wollen, denn er hat zu viele Mauselöcher und Mausefallen.' ${ }^{14}$

13 Vgl. Hans Franke, Spuren des Mesmerismus in der deutschen Literatur, in: Die Literatur, Jg. $37(1934 / 35)$, S. 89.

${ }^{14}$ Vgl. dazu Erman, a. a. O., S.17, Anm.1; die Stellenangabe für das Hufeland-Zitat 
Hufeland, derselbe Hufeland, der zweieinhalb Jahrzehnte zuvor der grimmigste Gegner Mesmers und des Magnetismus gewesen war, half in demselben Jahr 1809 entscheidend mit, die deutsche Medizin für den Mesmerismus zu gewinnen. «Die physische, nicht psychische Natur des Wirkenden» sei nun erwiesen, verkündete er, der angesehene Leibarzt, CharitéDirektor und Professor am Collegium medico-chirurgicum, in seinem «Journal der practischen Heilkunde». Hufeland magnetisierte seine Patienten zumeist nicht selbst. Er zog dafür den Chirurgen Carl Alexander Ferdinand Kluge heran. Kluge veröffentlichte zwei Jahre später ein Buch über den «animalischen Magnetismus als Heilmittel», das nicht weniger Aufsehen erregte als der vorangehende Aufsatz HufElands. Goethe hat es 1813 gelesen und sich Exzerpte daraus gemacht.

In diesen Jahren trat auch Wolfart auf den Plan. Er wurde 1810 an der neugegründeten Berliner Universität Privatdozent und wenige Jahre später Professor, sekundiert von dem romantischen Arzt und Dichter KoREFF, dem Günstling des Staatskanzlers Hardenberg. Wolfart machte Berlin zum Zentrum des Mesmerismus in der deutschen Medizin. Der Salon, in dem der kleine, zierliche und bewegliche Mann mit seinen dunklen «Feueraugen», seinem blassen Gesicht und seiner leisen Stimme den tierischen Magnetismus zelebrierte, wurde bald zu einem Treffpunkt des geistigen Berlin. Hier sehen wir Fighte, der - wie Schelling und Hegel die Erscheinungen des tierischen Magnetismus in sein philosophisches System einbezog und im magnetischen Fludium Mesmers «das letzte Objektive der Erscheinung» vermutete, Schleiermacher, Wilhelm und Caroline von Humboldt, sowie den Staatskanzler Hardenberg. Korefrss Einfluß auf ihn gründete sich auf den Mesmerismus.

Koreff gehörte zum Kreis um E.T.A.Hoffmann, der 1814 nach Berlin übergesiedelt war. In den Serapionsbrüdern Hoffmanns erscheint er als der «unruhige, unstete Vinzenz», als «der eifrigste Verehrer des Magnetismus». Vielfach kehren die Vorstellungen des Mesmerismus und der magnetische Schlaf in den Dichtungen Hoffmanns wieder, so im Kater Murr und in der Novelle Die Genesung, wie schon zuvor in seinem Magnetiseur.

Die Schriften von Wolfart und Kluge, von Gmelin und Wienholt über den Mesmerismus, sowie Schuberts Ansichten von der Nachtseite der Naturwissenschaften hatte auch JEAN PAUL sehr gründlich studiert und

bei Erman ist zu berichtigen in: Hufelands Journal der practischen Heilkunde, 79 (1834), I, S.47. Über Goethe und den Mesmerismus vgl. auch Tischner-Bittel, a.a.O., S. $139-45$ und $277-9$. 
Wolfart selber in Berlin kennengelernt. 1813 erschienen seine Muthmaßungen über einige Wunder des organischen Magnetismus in seinem «Museum», eine Abhandlung, in der die verschiedenen Aspekte des Mesmerismus naturwissenschaftlich, medizinisch und philosophisch diskutiert werden: das Sehen und Hören, der «Ätherleib», der Anteil des Gangliensystems, das Eisen, Magnetisieren durch Anblicken und Wollen, durch einen Spiegel, das magnetische Wasser, das Hellsehen, Magnetismus und Wahnsinn, Scheintod und Sterben, Aussichten ins zweite Leben. «Es ist», so beginnt erseine Abhandlung, «ein vollständiges Wunder, daß derselbeMagnet, welcher uns mit seiner Nadel die zweite Hälfte des Erdballs zeigte und gab, auch in der Geisterwelt eine neue Welt entdecken half.» ${ }^{15}$ Auch für JEAN PaUL ist also der tierische Magnetismus der Schlüssel zu den Grundfragen des Daseins ${ }^{16}$.

Jean Paul ließ es keineswegs bei der Theorie bewenden. Sehr anschaulich schildert ein junger kurländischer Arzt, Karl Bursy, der Jean Paul 1816 in Bayreuth besuchte, den Dichter als praktischen Magnetiseur: «Er hat selbst schon manchmal bei Zahn- und Kopfschmerzen seine Freunde mit Wirkung magnetisiert und wollte von mir wissen, ob er die Manipulation richtig vornehme. Ich mußte mich hinsetzen, und nun manipulierte er an mir in seinem Feuer so starken Druckes, daß es fast schmerzte. Ich bemerkte drauf, man müsse die Hände in einer geringen Entfernung vom Kranken halten. ,Ja, ja', sagte er, ,ich weiß wohl, ich will nur, daß Sie's fühlen, ob ich an den richtigen Stellen des Körpers den Zug leite.' Alles das geschah mit einer Schnelligkeit und Heftigkeit, die ich gar nicht beschreiben kann. Auf und ab lief er neben mir, riß mit einemmal das Fenster auf, schlug's dann wieder zu, knöpfelte den Rock zu und los, lief an den Tisch, wo unsere Gläser standen, die er angelegentlichst füllte, und machte manche interessante Bemerkung. ${ }^{17}$

${ }^{15}$ In gleichem Sinne äußerte sich Schopenhauer: «Der animalische Magnetismus ist, ... vom philosophischen Standpunkt aus betrachtet, die inhaltsschwerste aller jemals gemachten Entdeckungen» (zit. nach Tischner-BitteL, a. a. O., S. 264).

${ }^{16}$ Auch einen Schlüssel zum Verständnis der Geschichte glaubte man im tierischen Magnetismus gefunden zu haben. Görres erklärte die Anfänge der Menschheit als einen $\mathrm{Zu-}$ stand des Somnambulismus, des magnetischen Schlafes, als «ein Träumen in den tieferen Nervenzügen» (zit. nach Franke, a.a.O., S. 89). Man sah den «Abfall Tertullians durch somnambule Offenbarungen veranlaßt» (Arch. thier. Magn. 2, 1817, II, S. 160), man vermutete, daß Moнammed und andere Propheten oder die Jungfrau von Orleans nur «natürliche Somnambülen» gewesen seien (ebenda, 2, 1817, II, S. 161, und III, S. $130 \mathrm{ff}$.).

${ }_{17}$ Aus Kurländischen Reisetagebüchern, hrsg. von Otто Clemen (= Kurland in der Vergangenheit und Gegenwart, Bd. 7), Berlin-Steglitz, o. J., S. 58. 
Nicht nur Jean Paul magnetisierte, überall finden wir auch in diesen Jahren Laien als Magnetiseure. Im ganzen gesehen aber tritt das fachlichmedizinische gegenüber dem Laienelement jetzt stärker in Erscheinung. Die medizinische Literatur über den Mesmerismus wächst in die Breite. Kurz nacheinander entstehen zwei zentrale Zeitschriften, 1817 das von Eschenmayer in Tübingen, Kieser in Jena und Nasse in Halle herausgegebene «Archiv für den Thierischen Magnetismus», und 1818 die «Jahrbücher für den Lebens-Magnetismus oder neues Askläpieion», mit denen Wolfart sein altes «Askläpieion» wieder aufnahm. Dieses Schrifttum unterscheidet sich nicht nur durch seinen romantisch-naturphilosophischen Grundzug von der mesmeristischen Literatur der vorromantischen Zeit. Man ist nicht mehr in der Defensive gegen eine weit überlegene Zahl von Gegnern. Polemische Auseinandersetzungen, wie die «Bemerkungen über die Schrift eines verwegenen Finsterlings gegen den thierischen Magnetismus» im Eschenmayer-Kieser-Nasseschen Archiv fehlen auch jetztnicht. Aber sie treten in den Hintergrund gegenüber produktiven Beiträgen. Darunter verstand man vor allem Krankengeschichten und Berichte über Versuche mit Medien, die in langatmiger Breite die Veränderungen im Befinden eines magnetisch behandelten Patienten oder einer Versuchsperson über Wochen, Monate und selbst Jahre hin mit der umständlichen Treue eines Chronisten schildern. Dazwischen behaupten sich nur mühsam theoretische und philosophische Artikel oder Beschreibungen einzelner praktischer Verfahren.

Dabei bilden sich immer deutlicher zwei Richtungen heraus, von denen die eine durch den orthodoxen Mesmer-Anhänger und seinen von ihm selbst designierten Nachfolger WoLfaRT verkörpert wird, die andere, repräsentiert vor allem durch KIESER, einen mehr experimentell-naturwissenschaftlichen Kurs einzuschlagen sucht.

In den Rahmen dieses medizinischen Schrifttums, nicht in den der schönen Literatur, gehört auch Justinus Kerners so berühmt gewordenes Buch Die Seherin von Prevorst. Es ist bezeichnend für das Eindringen der Medizin in die Bezirke des Geistigen im Zeichen des Mesmerismus, daß die Literaturgeschichte dieses Buch mit aller Selbstverständlichkeit als ihr Objekt behandelte, und noch heute wandert es als ein längst traditionell gewordener Irrläufer von Literaturgeschichte zu Literaturgeschichte. In Wirklichkeit hat das Buch nichts mit der schönen Literatur zu tun. Es beginnt mit der Krankengeschichte von Friederike Hauffe, die Kerner für Jahre als Patientin in sein Arzthaus in Weinsberg aufnahm. Er behan- 
delte sie nicht nur, er bemühte sich mit ihrer Hilfe in unzählichen Versuchen, alle Phänomene des tierischen Magnetismus zu klären und zu sichern. «Reine Tatsachen» will KERNER hier geben ${ }^{18}$. Er hatte schon als elfjähriges Kind den Mesmerismus am eigenen Leibe kennengelernt. Im Bilderbuch aus meiner Knabenzeit schildert er, wie er, zuvor von dem robusten Brownianer WeIKard mit einem Getränk aus Tee, Eigelb, Kirschgeist und Pfefferkörnern gepeinigt, zu GMELIN in Heilbronn kam: «Er führte mich nun eine Treppe empor in ein kleines Zimmerchen . . . und hieß mich auf einen Stuhl setzen, sah mir mit seinen schwarzen Augen fest ins Auge und fing mich mit seinen ausgereckten Händen von Kopf bis in die Magengegend zu streichen an; er behauchte mir auch mehrmals die Herzgrube. Ich wurde ganz schläfrig und wußte endlich nichts mehr von mir. Ich mag lange schlafend gesessen sein, als ich erwachte ..., der Herr aber war nicht mehr da und ich sah ihn in meinem Leben nicht mehr ... In späteren Jahren begriff ich, daß mich der Herr magnetisiert hatte. ${ }^{19}$ Jetzt tat Kerner es GMELIN gleich. Zugleich suchte er, im Geiste eines KIESER forschend, naturwissenschaftliche Klarheit in die Welt des tierischen Magnetismus zu bringen und war doch allzu leicht bereit zu glauben. Mit unübertrefflicher Prägnanz hat Ricarda Huch die Gestalt Kerners und das Milieu, in dem er wirkte, gezeichnet: «Keiner von den Ärzten seiner Zeit ist so vom Zauber der Romantik umgeben wie Justinus Kerner, der Dichter, der Geisterseher, der, wenn er des Nachts zu Kranken gerufen wurde, von seinem Hündchen und von den Geistern der Verstorbenen, die er nicht hatte heilen können, begleitet, die wunderliche Runde machte. Wenn die Güte des Wollens und aufrichtige Hilfbereitschaft Grundbedingungen des magnetischen Wirkens sind, war Justinus KeRNER ausnahmsweise befähigt: er schien nur für andere da zu sein, sein Haus war stets voll von Hilfsbedürftigen aller Art, die das Vertrauen auf seine allbekannte rührende Herzensgüte und zum Teil auch das Gerücht von seinen magischen Kräften anzog. Von den nervösen und gemütsleidenden Freunden und von den Somnambulen abgesehen, hielten sich im Laufe der Zeit acht oder neun Besessene bei ihm auf, die scheu und bleich, unheimliche Erscheinungen, an den erschrockenen Kindern und Gästen vorüberstrichen. Sie wurden kakomagnetisch behandelt, das heißt die Dämonen, von denen der Kranke besessen war, wurden durch magnetische Manipulationen und die Überlegenheit eines guten Willens ausgetrieben. Eine solche Szene muß schauerlich, ja ent-

18 5. Auflage, Stuttgart 1877, S. 33.

19 Justinus Kerner, Das Bilderbuch aus meiner Knabenzeit, Wiesbaden 1948, S. 155 f. 
setzenerregend gewesen sein, da die Kranken wirklich wie von bestimmten Personen oder Teufeln bewohnt, die mit unnatürlichem Gebrüll aus ihnen heraus sprachen, sich gebärdeten, in den kritischen Augenblicken sich wehrten und in gräßlichen Krämpfen knirschend sich wälzten.» ${ }^{20}$ Und wo Mesmer die ätherischen Klänge der Glasharfe in den Dienst des Magnetismus gestellt hatte, griff Kerner zur Mundharmonika.

Als Kerners Seherin von Prevorst 1829 aus dem kleinen Landstädtchen hinaus in die Welt ging, war die Blütezeit des romantisch-naturphilosophischen Mesmerismus längst vorüber. KoREFF war in Ungnade gefallen, Wolfarts Praxis hatte aufgehört, eine der Sensationen Berlins zu sein, und seine Schüler und Anhänger hatten sich in mehrere einander befehdende Lager gespalten. Das spiegelt sich ebenso wie in der Medizin auch in der schönen Literatur wieder. Immermanns Novelle Der Karneval und die Somnambule, die 1830 erschien, ist aus dem gleichen Geiste spottender Ablehnung geschrieben, wie vier Jahrzehnte zuvor Thüмmels Schilderung des Straßburger Mesmeristenmilieus.

Es könnte scheinen, als sei ein Kreis geschlossen, als könne man alles, was zwischen Thümmel und Immermann liegt, in der Medizingeschichte einfach ausklammern als einen Irrweg, so wie das spätere 19. Jahrhundert, vom Fortschrittsglauben geblendet, die romantisch-naturphilosophische Medizin als einen nutzlosen, zeitvergeudenden Umweg betrachten zu können glaubte. Damit aber würde man den Tatsachen nicht gerecht. In der Tradition des Mesmerismus standen der Schotte BraID in Manchester, der ihn zum Hypnotismus umbildete, LiéBeault und Bernheim in Nancy, Charcot in Paris. Bei Charcot, Liébeault und Bernheim lernte wiederum Freud, dessen Weg zur Psychoanalyse von den Erfahrungen bei der hypnotischen Behandlung neurotischer Patienten ausging ${ }^{21}$. Und wie die Romantiker nicht nur um zu heilen den Weg zur «Nachtseite der Naturwissenschaften» gesucht hatten, sondern auch um zu erkennen, so bezogen Eduard von Hartmann, Klages und C.G. Jung in ihrer Philosophie und Psychologie des Unbewußten die gleiche Ausgangsposition. Jenseits von Wissenschaft und Medizin lebt die Zunft der «Magnetopathen» vom Erbe Mesmers. Wie sehr der Mesmerismus als historisches Phänomen auch noch die moderne Literatur zu faszinieren vermag, zeigen INa SEIDELS Wunschkind (1930) und Stefan Zweigs Heilung durch den Geist (1931).

${ }^{20}$ Ricarda Huch, Die Romantik, 2. Teil, 11./12. Auflage, Leipzig 1924, S. $281 \mathrm{f}$.

${ }^{21}$ Vgl. die Kapitel "From Mesmer to Freud" und "Freud and Modern Psychiatry" in: Iago Galdston, Progress in Medicine, New York und London 1940. 